\title{
Measurements of the CMS jet energy scale and resolution at $13 \mathrm{TeV}$
}

\author{
Milos Dordevic on behalf of the CMS Collaboration*广 \\ Vinca Institute of Nuclear Sciences, University of Belgrade \\ E-mail: milos.dordevicecern.ch
}

Jets are the experimental signatures of energetic quarks and gluons produced in high energy processes and they need to be calibrated in order to have the correct energy scale. A detailed understanding of both the energy scale and the transverse momentum resolution of jets at the CMS experiment is of crucial importance for many physics analyses. In this talk, we present the measurements of the CMS jet energy scale and resolution using the data sample collected in proton-proton collisions at a center-of-mass energy of $13 \mathrm{TeV}$.

ICHEP 2018, XXXIX International Conference on High Energy Physics

4-11 July 2018

Seoul, Korea

* Speaker.

${ }^{\dagger}$ A footnote may follow. 


\section{Introduction}

Jets are experimental signatures of quarks and gluons that are produced in high-energy processes such as proton-proton collisions at the LHC. Jet production cross-sections are several orders of magnitude higher than other processes. Particles like photons, electrons, muons and tau leptons can be mis-reconstructed as jets. The high event pileup presents a challenge to reconstruct and calibrate jets at the LHC. The measurements of jet energy scale and resolution using data sample recorded by the CMS experiment [1] at the center-of-mass energy of $13 \mathrm{TeV}$ are reported here.

\section{Jet reconstruction at the CMS experiment}

We identify several types of jets used at the CMS experiment, based on a different reconstruction algorithms. Particle-level jets are clustered from all stable $(\mathrm{c} \tau>1 \mathrm{~cm})$ and visible particles (excluding neutrinos). Jets reconstructed from energy deposits in calorimeter towers of electromagnetic and hadronic calorimeter, are referred as Calo jets. Lastly, the jets clustered from Particle Flow (PF) candidates combined with Charged Hadron Subtraction (CHS), the main algorithm used at the CMS experiment, and results in this report are based on this algorithm [2]. In this algorithm jets are reconstructed by clustering 4-momentum vectors of PF candidates, excluding those associated to pileup vertices.

\section{Jet calibration at the CMS experiment}

At the CMS experiment, jets are calibrated using a factorized approach that, on average, restores the jet energy scale to the particle jet level. The Fig. 1 shows a diagram of the calibration sequence, with each step applied to either MC or data. The L1 step, related to the pileup correction in MC and data, accounts for an offset energy coming from pileup. The L2L3 step applies the correction versus transverse momentum and pseudorapidity, as obtained from simulation. Lastly, the residual corrections are applied to data, to account for data to MC differences. Jet energy resolution is also measured, first on MC events and data to simulation scale factors are derived using dijet events.

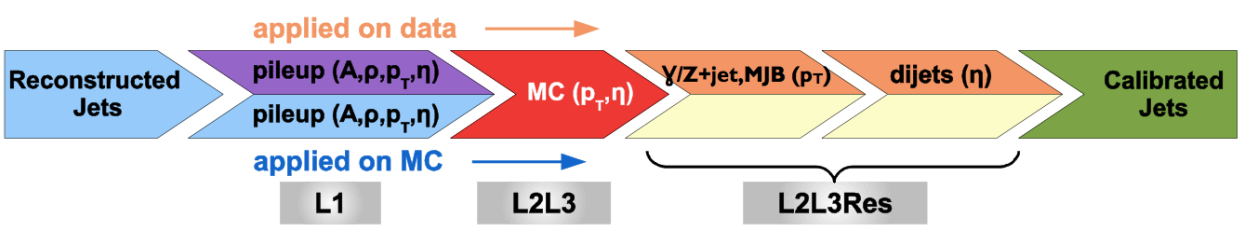

Figure 1: The sequence of steps applied for the jet energy correction procedure at the CMS experiment [4].

\subsection{Jet energy offset correction}

The residual offset pileup contribution, after the CHS, is subtracted in the L1 correction procedure. The offset is estimated using the Random Cone (RC) method [3] applied to Zero Bias data and Single Neutrino MC sample. The average transverse momentum of PF candidates in randomly placed cones, as measured from the RC method, indicates the average energy offset due to pileup. 
In the Fig. 2 the average offset per additional pileup interaction is presented for each type of PF particles: photons, EM deposits, neutral hadrons, hadronic deposits in HF and charged hadrons. The charged hadron deposits are further divided into two components: the ones associated with PU vertices that are removed by the CHS algorithm and the ones that are unassociated. The unassociated charged hadrons can not be removed from the list of PF particles by the CHS algorithm.

\subsection{Jet energy scale in simulation}

After the L1 offset step, the jet energy scale was restored to unity using simulated events. The jet response is shown in Fig. 3. The response is stable in the barrel region, with a drop for lower transverse momenta due to the limited HCAL acceptance. A stronger dependence on the jet transverse momentum is found in the endcap and forward region, as well as in the transition region.

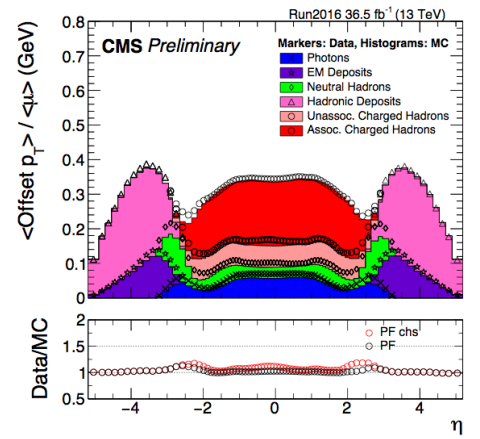

Figure 2: Random-cone offset measured in data and simulation, normalized by the average number of pileup interactions $\langle\mu\rangle$, separated by the type of PF candidates [4].

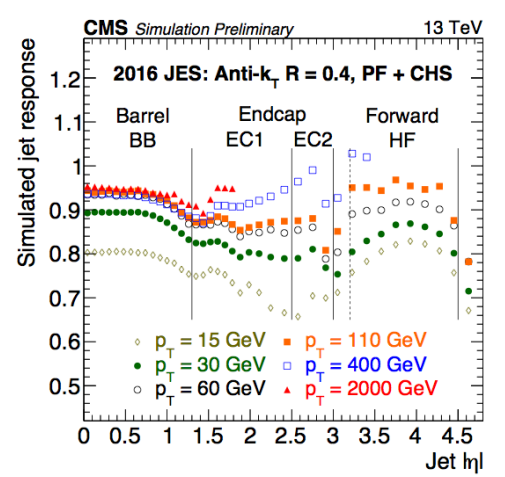

Figure 3: The jet response, defined as ratio of reconstructed and particle level jets, in bins of transverse momentum and pseudorapidity [4].

\subsection{Residual jet energy corrections}

To address residual differences between data and simulation, the additional scale factors are evaluated after correcting jets for pileup and simulated particle response. The basic idea is to exploit the transverse momentum balance between the jet to be calibrated and the reference object.

\subsubsection{Relative $\eta$-dependent corrections}

Relative residual corrections are derived as a function of transverse momentum and pseudorapidity, relative to the well calibrated detector region with $|\eta|<1.3$. The jet response is studied using transverse momentum balance and Missing Transverse Energy Projection Fraction (MPF) method with di-jet events. The nominal, MPF method, considers the response of whole hadronic activity in the event, recoiling versus the reference object and is less sensitive to ISR+FSR effects.

\subsubsection{Absolute $p_{T}$-dependent corrections}

The absolute jet energy scale corrections are determined with the $\mathrm{Z}(\mu \mu)+\mathrm{jets}$, the $\gamma+$ jets events and the multijet events. The response is obtained from the transverse momentum balance method. 


\subsubsection{Global fit of absolute residual corrections}

Absolute residual corrections are obtained from a simultaneous fit using three different events: $Z+j e t s, \gamma+j e t s$ and multijets, covering a wide range in the jet transverse momentum spectrum. Results of both pt balance and MPF methods for three data taking periods in 2016 are shown in Fig. 4. The solid line shows the global fit central value and the dotted lines are the statistical uncertainty of the fit. Scale uncertainties are treated as nuisance parameters in the fit and added quadratically to the chi-squared expression. The Run I results are included here as reference [3].
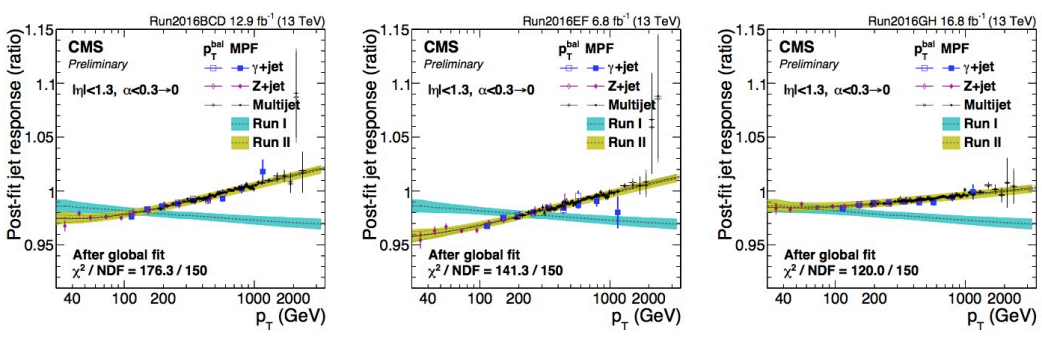

Figure 4: The absolute residual corrections, obtained combining the $\mathrm{Z}+\mathrm{jets}, \gamma+\mathrm{jets}$ and multijets events [4].

\subsection{Jet energy scale uncertainties}

The jet energy scale uncertainties, shown in Fig. 5, are derived versus transverse momentum and pseudorapidity and divided into sub-components: flat absolute scale uncertainties, the relative scale dijet uncertainties, the uncertainties due to pileup offset, the jet flavor uncertainties, the uncertainties due to differences in corrections per epoch and uncertainties due to method and sample.
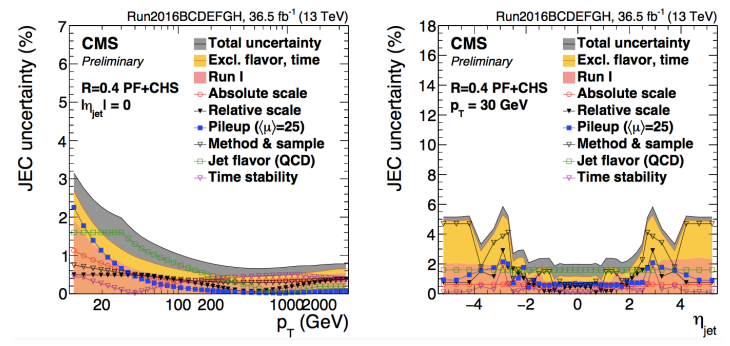

Figure 5: The total jet energy scale uncertainties with sources that are summed in quadrature [4].

\section{Summary}

Various data and MC samples were used to derive jet energy corrections at high precision, applying a factorized approach to cover a wide range of jet transverse momentum and pseudorapidity.

\section{References}

[1] The CMS Collaboration, JINST 3 S08004 (2008).

[2] The CMS Collaboration, JINST 12 P10003 (2017).

[3] The CMS Collaboration, JINST 12 P02014 (2017).

[4] The CMS Collaboration, CERN-CMS-DP-2018-028, http://cds.cern.ch/record/2622157. 